\title{
Algebraic Structures of Mathematical Foundations
}

\author{
Robert Murray Jones \\ University of Duesseldorf, Duesseldorf, Germany \\ Email: jones@uni-duesseldorf.de
}

How to cite this paper: Jones, R. M. (2020). Algebraic Structures of Mathematical Foundations. Open Journal of Philosophy, 10, 137-142.

https://doi.org/10.4236/ojpp.2020.101010

Received: December 31, 2019

Accepted: February 8, 2020

Published: February 11, 2020

Copyright (c) 2020 by author(s) and Scientific Research Publishing Inc. This work is licensed under the Creative Commons Attribution International License (CC BY 4.0).

http://creativecommons.org/licenses/by/4.0/

\begin{abstract}
In this paper, we continue to examine the role of algebra underlying geometry. The mathematics that we examine are abstract and are quite far removed from applications. Those who may wish to examine the possible uses of abstract geometry in physics might well begin by reading (Schlichenmaier, 2007; Bub, 1997). The method of research that we apply is to use the logic of H. S. Leonard to extract the underlying algebra of mathematical proofs and then use the results for the purpose of finding the algebra that underlies mathematical results.
\end{abstract}

\section{Keywords}

Rings, Factorization, Ideals, Polynomials

\section{Introduction}

In a previously published paper (Jones, 2014), we emphasized the use of a system of logic devised by Henry Siggins Leonard to facilitate the analysis of logical and mathematical structures. In this paper we shift the emphasis from that logic, upon which we continue to rely, to the use of algebra in mathematics. We wish to particularly draw the reader's attention to recent literature in closely related subject areas, e.g. (Murphy, 1990) is highly recommended as a textbook of this subject.

\subsection{Algebraic Structures}

Here we examine several fundamental algebraic structures that frequently are used in mathematical writing and proofs.

\subsection{Some Preliminaries}

A certain theorem, named after Bézout (Bézout, 2006), is a criss-cross point of at 
least three of mathematics. These are algebraic geometry, polynomials, and projective geometry. We wish to examine the underlying algebra involved in this result. In doing so, we follow the original reasoning published by Bézout, but we mention several mathematical developments, that have appeared in the meantime, that are relevant to his result.

\subsection{Elementary Algebraic Structures}

A group consists of a set $G$ and an operation $\circ$. If for all elements $x$ and $y$ of $G$, the item $z=x \circ y$ is also an element of $G$, then $G$ is said to be closed with respect to $\circ$.

There are three conditions that define a group.

1): If $x, y$, and $z$, are elements of $G$, then $(x \circ y) \circ z=x \circ(y \circ z)$. $\circ$ is associative.

2): There is an element $e$ of $G$ such that $e \circ x=x \circ e=x$ There is an identity element $e$ in $G$.

3): For every element $x$ of $G$ there is an element $x^{-1}$ of $G$ such that $x \circ x^{-1}=x^{-1} \circ x=e$. Each element of the group has an inverse.

David Hilbert made a fundamental suggestion that has since permeated mathematics. He suggested first-order logic with a set theory for the formulation of the foundations of mathematics (Moore, 2002). We introduce some of the notations of Hilbert's initiative, although we do so with a somewhat different purpose than he had.

Universal and existential quantifiers, and set theory membership,

$$
\forall x \in \mathbb{R}, \exists y \in \mathbb{R}
$$

Conjunction, disjunction, negation, implication, $\wedge, \vee, \neg, \rightarrow$

The notation of the above definition of a group can now be augmented. A group consists of a set $G$ and an operation $\circ$. Three conditions define a group.

$$
\exists e \in G \wedge \forall x \in \mathbb{G} \wedge \forall y \in \mathbb{G} \wedge \forall z \in \mathbb{G}
$$

1) $(x \circ y) \circ z=x \circ(y \circ z)$

2) $e \circ x=x \circ e=x$

3) $\exists x^{-1} \in G, x \circ x^{-1}=x^{-1} \circ x=e$

We introduce here notational conventions of set theory and logic in the style of Hilbert and proof structure in the natural deduction style of Gentzen. These gentlemen provide us with an assemblage of methods for bringing to light the underlying algebraic structure of the mathematical results to which these methods lend proofs. The interested reader may wish to consult (Pelletier, 1999) for the history of these techniques.

Another elemental algebric structure that is of central importance for understanding geometry is that of a ring. A ring $R$ contains two opertors: multiplication $\cdot$ and addition + .

$$
\exists 0 \in R \wedge \forall x \in \mathbb{R} \wedge \forall y \in \mathbb{R} \wedge \forall z \in \mathbb{R}
$$

1) $x+y=y+x$ 
2) $(x+y)+z=x+(y+z)$

3) $x+0=x$

4) $\exists(-x) \in R, \quad x+(-x)=(-x)+x=0$

5) $(x \cdot y) \cdot z=x \cdot(y \cdot z)$

6) $(y+z) \cdot x=y \cdot x+z \cdot x$

7) $x \cdot(y+z)=x \cdot y+x \cdot z$

The axioms for a ring can be usefuly thought of as falling into three sets. Axioms 1 to 4 , for the addition operator, characterize the set $R$ as a group. The group operator is commutative and is, for this reason, often called Abelian. Axiom 5 asserts that multiplication is associative. The third, and last, subset of axioms for a ring are 6 and 7. These axioms relate the two ring operators to each other, and are often called distribution operators.

A subring of a ring $R$ is a nonempty subset $S$ of $R$ that is also a ring with the same operators for multiplication and addition as $R$.

Next, we need the concept of an ideal. A subring $A$ of a ring $R$ is said to absorb elments of $R$ if the product of any element a of $R$ and any element $b$ of $A$ is in $A$. As an example, the even integers are a subring of the integers that absorb elements of the ring of integers. This is so, because the product of any integer with an even integer is an even integer. An ideal is a subring that absorbs elements of the ring.

\subsection{The Concept of Coset}

Cosets: A concept that is very useful is that of a coset. If $S$ is a subgroup of the group $G$ and $a$ is an element of $G$ then the notation $a S$ is written to indicate the set of all products of a with elements of $S$.

The concept of a coset also lends itself to definition using a notation of algebra and set theory that is sometimes described as the circumflex notation.

$$
a S:=\hat{x}(\exists y)(y \in \mathbf{S} \wedge(x=a \cdot y))
$$

This definition is intended to be read as: the expression consisting of a lower case a concatenated with an upper case $S$ is by definiton equal to the set of those $x$ such that there is an element $y$ of the subring $S$ such that $x$ is equal to the ring product of $a$ with $y$.

\subsection{The Role of a Determinant}

We now can return to the proof of the Theorem of Bézout as documented in (Bézout, 2006), Although we intend, in what follows, to follow the methods of proof reasoning published by Bézout, we deviate from the assertions of that proof themselves. Many of those proof assertions, since his time, have been incorporated into the structure of matrices and determinants. We propose to use the latter structures to express the proof that we now continue to examine.

At this point, we define and make use of a determinant. This determinant was discovered by James Joseph Sylvester (Sylvester, 1973). 


$$
\operatorname{det}=\left[\begin{array}{cccccccc}
c_{1} & c_{2} & \cdots & c_{m} & 0 & 0 & \cdots & 0 \\
0 & c_{1} & c_{2} & \cdots & c_{m} & 0 & \cdots & 0 \\
0 & 0 & c_{1} & c_{2} & \cdots & c_{m} & 0 & 0 \\
\vdots & & & & & & & 0 \\
0 & 0 & \cdots & c_{1} & c_{2} & c_{3} & \cdots & c_{m} \\
d_{1} & d_{2} & \cdots & d_{n} & 0 & 0 & \cdots & 0 \\
0 & d_{1} & d_{2} & \cdots & d_{n} & 0 & \cdots & 0 \\
\vdots & & & & & & & 0 \\
0 & 0 & \cdots & d_{1} & d_{2} & d_{3} & \cdots & d_{n}
\end{array}\right]
$$

The above determinant is useful because it illuminates a result first obtained by the French mathematician Étienne Bézout (Bézout, 2006). It does, however, replace explicit use of the underlying algebra of this result to bring that result to light, with the charming symmetry of the matrix of the determinant. The determinant belongs to applied mathematics, in which the Theorem of Étienne Bézout, also has its place. The regularity of the array, in rows and columns, also lends itself to computer applications.

This is determinant also plays a role in a part of the proof of the Theorem of Bézout. The most subtle part of that proof deals with a so-called multiplicity of the intersection points of curves. The above determinant will help us to deal with this problem. We must, however, postpone this part of our discussion until we have developed the needed preliminaries.

For the reader who may wish to read a review of the considerations outlined above, we recommend (Sharpe, 1987). The Theorem of Bézout has many incarnations in many contexts. The preferred such context is not the classical Euclidean plane, but rather the projective plane. The reader may take a look at what is to come by examining this geometric context in (Apéry, 1987). This beautiful book first describes the geometry of the projective plane, then shows diagrams of the geometric constructs, and then offers color plates of those geometric constructs.

\subsection{Algebraic Geometry}

Having called the reader's attention to these matters, we wish now to continue to examine the underlying algebra of the fundamental result of Étienne Bézout. This examination will lead us in the direction of algebraic geometry.

We hope to continue this line of inquiry in another paper. The plan of our exposition is to discuss this very large field of mathematical work in a series of consecutive papers, each of which constitutes a small part to a larger discussion of the many aspects of the Theorem of Étienne Bézout. We also look forward to discussing the applications of algebra to physics, as mentioned in the Abstract. We also look forward to describing the uses of algebra in Morse theory and to algorithms for the analysis of data. These are described in (Knudson, 2015).

\section{Conclusion}

We have begun to compare various approaches to proofs of the Theorem of 
Étienne Bézout. Some of these approaches are in the following literature references. (Gibson, 1998) offers a well-written formulation of a standard interpretation of the theorem. (Kendig, 2015) uses an interesting recursive approach to the proof.

We are using the methods described in Section 1 to find shared underlying algebraic methods underlying these various approaches to the proof.

\section{Acknowledgements}

We wish to express our indebtedness and thanks to two students of Henry Leonard for their numerous recollections of his teachings in logic. They are: Joanne Eicher (University of Minnesota, Twin Cities) and Rolf George (University of Waterloo, Ontario). We are indebted to Henry S. Leonard for his paper (Leonard, 1969). We wish to thank Craig Smoryński, for calling (Hartshorne, 1997) to our attention. It contains an invaluable historical review of the development of axiomatic method in mathematics.

\section{Conflicts of Interest}

The authors declare no conflicts of interest regarding the publication of this paper.

\section{References}

Apéry, F. (1987). Models of the Real Projective Plane. Braunschweig: Vieweg. https://doi.org/10.1007/978-3-322-89569-1

Bézout, É. (2006). General Theory of Algebraic Equations. Princeton, NJ: Princeton University Press. (Translated by Eric Feron from Theorie général des équations algébrique, Paris, 1770)

Bub, J. (1997). Interpreting the Quantum World. Cambridge: Cambridge University Press.

Gibson, C. (1998). Elementary Geometry of Algebraic Curves. Cambridge: Cambridge University Press. https://doi.org/10.1017/CBO9781139173285

Hartshorne, R. (1997). Geometry: Euclid and Beyond. New York: Springer Verlag.

Jones, R. M. (2014). Model Theories of Set Theories and Type Theory. Open Journal of Philosophy, 4, 54-58. https://doi.org/10.4236/ojpp.2014.41008

Kendig, K. (2015). Elementary Algebraic Geometry (2nd ed.). Chicago, IL: Dover.

Knudson, K. P. (2015). Morse Theory, Smooth and Discrete. Hackensack, NJ: World Scientific. https://doi.org/10.1142/9360

Leonard, H. S. (1969). The Logic of Existence. Philosophical Studies, 7, 49-64. https://doi.org/10.1007/BF02221764

Moore, G. H. (2002). Hilbert on the Infinite, the Role of Set Theory in the Evolution of Hilbert's Thought. Historia Mathematica, 29, 40-64. https://doi.org/10.1006/hmat.2001.2332

Murphy, G. (1990). C $C^{*}$-Algebras and Operator Theory. San Diego, CA: Academic Press.

Pelletier, F. J. (1999). A Brief History of Natural Deduction. History and Philosophy of Logic, 20, 1-31. https://doi.org/10.1080/014453499298165 
Sharpe, D. (1987). Rings and Factorization. Cambridge: Cambridge University Press. https://doi.org/10.1017/CBO9780511565960

Sylvester, J. (1973). The Collected Mathematical Papers of James Joseph Sylvester (Volume I (1837-1853), pp. 247, 263, 249). New York: Chelsea Publishing Company.

Schlichenmaier, M. (2007). An Introduction to Riemann Surfaces, Algebraic Curves and Moduli Spaces. Berlin: Springer. 